\title{
El peso del origen social y del centro escolar en la desigualdad de resultados al final de la escuela obligatoria*
}

\author{
Dani Torrents \\ Rafael Merino \\ Maribel Garcia \\ Ona Valls
}

Universitat Autònoma de Barcelona. Departament de Sociologia

danitv@hotmail.com; rafael.merino@uab.cat; maribel.garcia@uab.cat; onavalls@gmail.com

Recepción: 09-03-2016

Aceptación: 28-10-2016

\section{Resumen}

El debate sobre el efecto de los centros escolares ha sido ingente desde la aparición del informe Coleman. La desigualdad de rendimiento educativo entre el alumnado según el centro escolar al que acude es un hecho conocido tanto por científicos sociales como por muchas familias, que lo tienen en cuenta en sus estrategias de elección de escuela. La cuestión clave es dirimir si esta diferencia se debe más al efecto composición o si en ella interviene la acción pedagógica del centro. Como indicador de acción pedagógica se ha escogido la comprensividad en el currículo. La hipótesis que se plantea es que el efecto de composición es mayor que la organización del currículo. Los datos que se han utilizado provienen de una encuesta realizada a 1782 estudiantes que cursaban $4 .^{\circ}$ de ESO en 27 centros de la ciudad de Barcelona. Se ha utilizado un modelo de análisis lineal jerárquico para medir las diferencias entre centros controlando las características individuales de los alumnos. Las diferencias entre centros se atribuyen fundamentalmente a la composición social del alumnado, y con un carácter residual al currículo más o menos comprensivo. También se destaca que el efecto composición tiene un impacto menor en las chicas de clase baja, ya que obtienen mejores resultados de los esperados en centros con mayor complejidad social.

Palabras clave: comprensividad; composición social; modelo multinivel; sexo; rendimiento; logro escolar

* Esta investigación tiene el apoyo del Plan Nacional I+D (referencia CSO2013-44540-P). 
Abstract. Social background and school effect in performance inequalities at the end of compulsory education

School effects have been a controversial issue since the publication of the Coleman report. Students' attainment varies depending on the school they attend; a fact that is well known in social scientific research as well as in families, who take these differences into account in their school choice strategies. The key issue is whether this difference is related to the student social composition or to the pedagogical model implemented by the school and based on the comprehensive curricula. The main hypothesis is that the impact of social composition is greater than that of comprehensiveness. Data were collected from 1,782 secondary school students in the 10th grade at 27 schools in the city of Barcelona. A hierarchical linear model is run to analyze the differences between schools, controlling for the individual features of students. The differences between schools are mainly due to the social composition of students and the different degrees of comprehensiveness is not relevant. Moreover, lower class female students are less exposed to the composition effect because they perform better than expected in more socially complex schools.

Keywords: comprehensive school; social composition; multilevel model; sex; academic performance; school achievement

\section{Sumario}

1. Introducción. El papel de los centros escolares en las desigualdades educativas

2. Desigualdades en el rendimiento escolar. Origen social, composición social y comprensividad
3. Modelo de análisis

4. Análisis de resultados

5. Conclusiones

Referencias bibliográficas

Anexo

\section{Introducción. El papel de los centros escolares en las desigualdades educativas}

Desde la aparición del llamado informe Coleman (1966), es ingente la literatura sociológica dedicada al análisis de las desigualdades de resultados educativos y el peso de los factores sociales asociados a estas desigualdades (para una revisión, ver Carabaña, 2016). Una de las conclusiones más controvertidas de este informe fue que los factores estrictamente escolares (de organización curricular o de modelo pedagógico) tenían muy poca incidencia en las desigualdades de resultados educativos. $\mathrm{O}$, por decirlo de forma más precisa, había más variabilidad dentro de cada escuela que entre tipos organizativos y pedagógicos de escuelas. Eso quería decir que el peso de la explicación debía buscarse en otros factores asociados a las características de las familias, bien por la relación directa de la socialización familiar, bien por la relación indirecta de escoger una escuela selectiva socialmente. Estos resultados desconcertaron tanto a investigadores como a reformistas, los que impulsaban y confiaban en cambiar los métodos 
didácticos para reducir las desigualdades y los que impulsaban políticas para acabar con la segregación escolar, que en la década de 1960 en los Estados Unidos tenía un fuerte contenido racial (Fernández Enguita, 2016).

El objetivo del presente artículo es contribuir a promover el debate sobre el papel de los centros docentes en las desigualdades de los resultados escolares, en concreto la organización del currículo en el último curso de la educación obligatoria. La hipótesis principal, siguiendo a Coleman et al. (1966), es que la composición social del alumnado de los centros tiene mayor importancia que la organización del currículo y la separación por niveles a la hora de explicar los resultados escolares, al pesar más los elementos estructurales que los pedagógicos. A menudo el debate académico y educativo se centra en la organización del currículo más o menos comprensivo sin tener en cuenta el entorno de los centros y la composición social de los mismos. Se obviaría, pues, que lo estructural puede condicionar tanto el desarrollo como los resultados de lo pedagógico. Por este motivo, nuestro objeto de estudio se centra en el análisis de esta composición, para analizar si las diferencias de resultados educativos de los alumnos se deben a características individuales, a la concentración de estudiantes que muestran una característica determinada o a las prácticas de agrupación de alumnos por habilidades. Además, se propone analizar hasta qué punto el papel de los centros escolares en estos resultados varía en función de las características individuales de cada alumno y, por lo tanto, la relación que se produce entre el nivel individual y el nivel de la institución docente en general.

Para ello, en el primer apartado se sitúan las principales aportaciones teóricas respecto a la relación entre origen social y rendimiento, poniendo énfasis en las que se aborda desde el marco de las variables escolares. En el segundo se explica el modelo de análisis a seguir, las variables y la fuente de los datos utilizados. El tercer apartado muestra los resultados empíricos del modelo. Finalmente, en el apartado de conclusiones abordamos las implicaciones de los resultados para la política educativa, con el objetivo de reflexionar sobre la eficacia de las medidas que tienen como objetivo la reducción de las desigualdades escolares y sociales.

\section{Desigualdades en el rendimiento escolar. Origen social, composición social y comprensividad}

El campo de las desigualdades educativas es muy amplio, y opera de forma distinta en función del momento (acceso, proceso o resultado) y del nivel (escuela preobligatoria, obligatoria o postobligatoria) (Merino, 2012). Nuestra investigación se enmarca en el estudio de las desigualdades de resultados de la escuela obligatoria, relacionándolas con elementos de acceso y de proceso dentro de la escuela. Como detallaremos en el modelo de análisis, el acceso tiene que ver con el efecto composición determinado por la elección de escuela por parte de las familias; el proceso, en cambio, se relaciona con el tipo de organización curricular, que en nuestro caso se concreta en el último curso de escolarización obligatoria. 
A partir de la constatación empírica de una relación existente entre los resultados escolares y el origen social de los alumnos, la sociología ha desarrollado numerosas investigaciones para encontrar los factores que expliquen dicha circunstancia. La mayoría de estas investigaciones se enmarcan dentro de las corrientes llamadas de la reproducción social, que atribuyen al capital económico, cultural, simbólico o lingüístico de las familias el efecto causal para explicar por qué los hijos de las clases populares tienen menor éxito en el logro académico. En los últimos años esta perspectiva se ha visto reforzada por los conocidos informes PISA y la renovada constatación de la relación entre las competencias que mide la OCDE y el estatus socioeconómico de las familias (Ferrer, 2011; Dupriez et al., 2008). Desde esta perspectiva, la distancia entre logro escolar y origen social estaría mediada, cuando no reforzada, por unas prácticas institucionales (organizativas y pedagógicas) selectivas, sea en el acceso (elección de escuela, distinción entre escuela pública y escuela privada) o en el proceso (efecto de expectativas confirmadas o efecto Pigmalión, agrupación de alumnos por nivel de rendimiento, clima escolar, etc.). En OECD (2013) se puede encontrar una revisión al respecto.

Desde el punto de vista empírico, hay que decir que las teorías de la reproducción explican razonablemente bien la media, pero no explican o no ayudan a entender la desviación típica. Es decir, existe abundante evidencia de que las probabilidades de tener mayor éxito escolar aumentan cuanto mayores son los niveles de instrucción de los progenitores (Carabaña, 1993; Calero, 2007; Forquin, 1985; Jackson et al., 2007), pero en general se trata de correlaciones débiles o muy débiles, que responden a una considerable variabilidad interna dentro de cada clase social o de cada nivel de instrucción familiar. Esta variabilidad se pone de manifiesto en el diferencial de logro educativo de los hijos de clase trabajadora. El hecho de que haya un porcentaje significativo de estos adolescentes que obtienen buenas notas hace que tengamos que elaborar explicaciones, algunas ad hoc, para entender esta «desviación» respecto a la media de su grupo.

Una explicación que ha cobrado fuerza en los últimos años es la hipótesis de la resiliencia. Es decir, la hipótesis de que estos jóvenes tienen una mayor capacidad de superación que sus coetáneos ante un entorno hostil o de privación relativa. Otra explicación plausible, pero que la sociología es reacia a incorporar, es la de la inteligencia ${ }^{1}$. Cabrera y Cabrera (2008) identifican una correlación más fuerte del rendimiento escolar con un indicador del cociente de inteligencia que con el nivel educativo de los padres, aunque también es cierto que a mayor nivel educativo de los padres mayor cociente de inteligencia presentan sus hijos e hijas. La explicación que sostienen los autores es que el test que mide la inteligencia (realizado por alumnos de 15 años) no mide la inteligencia "natural», sino que también es fruto de la socialización primaria de los padres.

1. La definición de la inteligencia es un campo minado. Pocos sociólogos se han atrevido a medirla y a incorporarla en la relación entre origen social y rendimiento. 
Así como dentro de cada grupo social hay diferencias en las aptitudes físicas, también podemos contemplar la posibilidad de que existan aptitudes cognitivas distintas ${ }^{2}$, y parece razonable pensar que las aptitudes cognitivas tienen relación con el aprendizaje escolar $y$, por lo tanto, con los resultados académicos (Carabaña, 2012). Introduciendo la aptitud como variable intermedia entre origen social y resultados académicos, se hace innecesario recurrir a la resiliencia como factor explicativo del buen resultado escolar de alumnos de familias con escasos recursos económicos o culturales (Carabaña, 2016).

Otro elemento a tener en cuenta es la socialización familiar. A menudo se tiende a confundir clase social y familia (Carabaña, 1993), cuando efectivamente existen estilos de socialización diferentes dentro de cada clase social. La distribución de roles en el entorno familiar (por ejemplo: un reparto equitativo de las tareas domésticas) y la valoración del esfuerzo por el aprendizaje o la motivación familiar pueden ser la clave para el desarrollo de competencias que facilitan el éxito escolar a igualdad de origen de clase social (Martín Criado et al., 2000). Finalmente, no hay que descartar la variabilidad debida a otros factores no conocidos y/o difíciles de conocer $^{3}$ (ver, por ejemplo, Jackson, 2013), que es lo que estadísticamente se denomina el residuo.

Estas aportaciones son algunas de las que proponen explicaciones en las diferencias de rendimiento, tanto entre orígenes sociales distintos como dentro de un mismo grupo social, pero además las hay que ponen el foco en el centro docente. Los valores familiares también pueden influir a la hora de elaborar estrategias y elecciones educativas que pueden alterar los resultados esperados en la correlación entre origen social y logro académico. Algunas familias de clase trabajadora buscan escuelas en entornos de clase media con la creencia de que garantizan unos mejores resultados. Es decir, esperan que sus hijos se asemejen más a los escolares de clase media siempre que el porcentaje de alumnos de clase baja sea menor, con lo que se refuerza la segregación colegial (Bonal, 2012a) y el efecto composición.

Esta semejanza podría darse por un mecanismo de interacción entre pares (Carabaña, 2016; Harris, 1999), de contagio de expectativas (Subirats, 2008) o bien por un mecanismo de autoselección dentro del grupo minoritario de cada contexto, fenómenos que también se dan en la enseñanza superior (Troiano y Elias, 2014). También hay que destacar que puede producirse como efecto contrario el cierre social de algunas escuelas a estos grupos subalternos ${ }^{4}$, en

2. No estamos hablando de alteraciones que condicionan el aprendizaje y que necesitan adaptaciones curriculares o en algunos casos escolarización diferenciada.

3. Dejamos de lado factores que pueden ser muy importantes pero escasos en nuestro contexto socioeconómico, como la malnutrición, el elevado número de hermanos o las deficiencias afectivas en la primera infancia. El aumento de la pobreza infantil en los últimos años también puede tener un efecto potencial y a largo plazo en el desarrollo de las capacidades cognitivas y, por lo tanto, en el logro escolar.

4. Precisamente, una de las constataciones del informe Coleman fue la llamada White Flight de familias blancas de clase media, cuando vieron que hijos de familias negras de clase baja se repartían por las escuelas de entornos de clase media como consecuencia de la promulgación de leyes de derechos civiles y contra la discriminación. 
nuestro contexto con el aumento de costes indirectos de la escolarización en numerosos centros privados concertados e incluso en algunos públicos (Bonal, 2012b).

Por último, las teorías de la reproducción social atribuyen a la escuela un papel perpetuador, e incluso amplificador, de las desigualdades sociales a través de dos mecanismos que actúan en el proceso y que hemos mencionado anteriormente: el profesorado y el currículo. Hay un punto en común entre ambos y que es pertinente para nuestra investigación: la separación de alumnos por nivel de competencia o rendimiento y la atribución del profesorado en la evaluación y la asignación de los alumnos a los distintos grupos.

En este sentido, las prácticas pedagógicas selectivas tenderían a reproducir las expectativas y los resultados esperados, puesto que se concentrarían alumnos de origen social más alto en los grupos de mejor nivel y, al contrario, a concentrar alumnos de origen social más bajo en los grupos de menor nivel, con más adaptación curricular, más rezagados, etc. La evidencia empírica de esta diferenciación es abundante, pero no concluyente (Fernández Enguita, 2014). Una de las razones es que de nuevo se confunde rendimiento con aptitud (Carabaña, 2012), y aunque es cierto que en los grupos de nivel bajo se acumulan estudiantes de minorías étnicas y/o de origen humilde (Pàmies y Castejón, 2015) por mostrar unos resultados escasos, no sabemos si esos resultados se deben a sus aptitudes o a la percepción de los profesores respecto al rendimiento esperado.

En todo caso, durante las últimas décadas, se han impulsado las llamadas reformas comprensivas para reducir las prácticas selectivas (o segregadas, si se quiere acentuar su connotación negativa) y aumentar las prácticas de agrupación heterogénea con el objetivo de reducir la discriminación. Pero la aplicación de estas prácticas difiere mucho en función del contexto donde se ubica la escuela y la composición social de su alumnado (Benito y González, 2013). No hay unanimidad en la literatura internacional sobre los efectos de la comprensividad o segregación del alumnado en los resultados escolares. Hay estudios que apuntan a una mayor desigualdad de resultados cuanto más temprano es el tracking (Hanushek y Woessman, 2006; Duru-Bellat y Mingat, 1997). En cambio, otros estudios encuentran un efecto más bien leve en la agrupación por niveles en los resultados (Hattie, 2009; Slavin, 1990).

El estudio quizás más preciso sobre la relación entre comprensividad, rendimiento y clase social se realizó con datos longitudinales a partir del National Child Development Study (Glaeser y Cooper, 2012). Estos autores compararon distintos distritos ingleses en los años sesenta, algunos habían aplicado la reforma comprensiva y otros no. Comprobaron que a los chicos de clase baja pero con altas habilidades (detectadas mediante un test que hacían a los 11 años) obtenían mejores resultados en las escuelas selectivas. Pero más recientemente, y con datos PISA, otro estudio comprueba que estos resultados podrían ser debidos precisamente al efecto composición. Si se toma en consideración dicho efecto, la agrupación selectiva del alumnado deja de tener consecuencias en el rendimiento (Benito y González, 2013). 
En definitiva, existe una gran complejidad en la relación entre origen social, centro escolar y rendimiento educativo, pero a su vez representa un campo de análisis interesante para el contraste teórico y relevante para la política educativa. En el siguiente apartado se describe el modelo de estudio y los principales resultados de la investigación llevada a cabo en la ciudad de Barcelona en 27 centros públicos y privados concertados, de composición social desigual.

\section{Modelo de análisis}

Nuestro modelo de análisis establece las relaciones entre la clase social y el rendimiento escolar de los jóvenes de nuestro estudio, en función de las características de los centros en los que estudian $4 .^{\circ}$ de ESO. Nuestra pregunta de investigación se plantea si el alumnado de clase baja obtiene mejor rendimiento en los centros con heterogeneidad social y/o en los centros que apuestan por una secundaria más comprensiva o menos selectiva, a fin de aportar resultados contrastados al debate científico presentado en el epígrafe anterior.

Los datos proceden de una investigación más amplia de carácter internacional, en la que se comparan muestras de jóvenes en el décimo grado de escolaridad obligatoria de doce ciudades de países diferentes (ISCY Project ${ }^{5}$ ), sus características, expectativas y trayectorias después de la escuela obligatoria. Para realizar el presente artículo, utilizamos datos primarios recogidos durante la elaboración de la primera ola del estudio longitudinal, llevado a cabo entre febrero y abril de 2014 en la ciudad de Barcelona. La muestra es de 1.782 estudiantes que cursaban $4 .^{\circ}$ de ESO en el año académico 2013-2014 matriculados en 27 centros públicos y privados concertados. Los centros están escogidos a partir de una muestra estratificada en función de la titularidad y de la complejidad social del entorno, con el objetivo de poder disponer de representatividad del conjunto de alumnos de la ciudad (tabla 1).

Tabla 1. Estadísticos descriptivos de la variable dependiente

\begin{tabular}{|c|c|c|c|c|c|c|}
\hline & \multicolumn{2}{|c|}{ Centros públicos } & \multicolumn{2}{|c|}{ Centros concertados } & \multicolumn{2}{|r|}{ Total } \\
\hline & $\begin{array}{c}\text { Centros } \\
n\end{array}$ & $\begin{array}{c}\text { Alumnado } \\
n(\%)\end{array}$ & $\begin{array}{c}\text { Centros } \\
n\end{array}$ & $\begin{array}{c}\text { Alumnado } \\
n(\%)\end{array}$ & $\begin{array}{c}\text { Centros } \\
n\end{array}$ & $\begin{array}{c}\text { Alumnado } \\
n(\%)\end{array}$ \\
\hline Alta complejidad & 4 & $316(15,37)$ & 4 & $163(7,93)$ & 8 & $479(23,3)$ \\
\hline Complejidad media & 6 & $517(25,15)$ & 8 & $782(38,04)$ & 14 & $1.299(63,18)$ \\
\hline Baja complejidad & 2 & $93(4,52)$ & 3 & $185(9)$ & 5 & $278(13,52)$ \\
\hline Total & 12 & $926(45,04)$ & 15 & $1.130(54,96)$ & 27 & $2.056(100)$ \\
\hline
\end{tabular}

Fuente: elaboración propia a partir de datos del ISCY Project.

Respecto a la titularidad, se ha tenido en cuenta si se trataba de centros públicos o concertados. Los centros privados no concertados no constan en el estudio por su escaso peso respecto al total y por la dificultad de acceder a ellos

5. Para más información, consultar la web: http://iscy.org/ 
Tabla 2. Estadísticos descriptivos de la variable dependiente

\begin{tabular}{lc}
\hline & Rendimiento \\
\hline Media & 74,9 \\
Mediana & 78,0 \\
Desviación típica & 14,9 \\
Mínimo / Máximo & $16,6 / 100$ \\
\hline
\end{tabular}

Fuente: elaboración propia a partir de datos del ISCY Project.

durante el trabajo de campo. Respecto a la complejidad del centro, se ha tenido en cuenta un índice diseñado por la Administración docente que incorpora diversos indicadores del alumnado con problemáticas educativas e indicadores del entorno socioeconómico de los centros.

La variable dependiente es el rendimiento académico, medido por el resultado de las pruebas de competencias básicas proporcionado por el Consejo Superior de Evaluación del Sistema Educativo de Cataluña. Son pruebas que se realizan a todos los alumnos matriculados en $4 .^{\circ}$ de ESO, realizadas de forma externa a los centros y que muestran una finalidad diagnóstica de las competencias y de los conocimientos lingüísticos y matemáticos que los alumnos han adquiridos en el último año de la educación obligatoria. De todas las pruebas de competencias que se realizan, hemos escogido como indicador sintético la media aritmética de la prueba de matemáticas y de lengua catalana. El rango de puntuación va de 0 a $100^{6}$, y estos son los descriptivos básicos (tabla 2):

Como indicador de clase social se ha utilizado la categoría profesional de los padres $^{7}$, codificando las respuestas de los alumnos en ISCO- $08^{8}$ y adaptándolas posteriormente al esquema EGP (Erikson y Goldthorpe, 1992). Una vez obtenidas las siete categorías de EGP, se han agrupado del siguiente modo:

a) Empresarios y profesionales (I + II).

b) Pequeños propietarios y trabajadores no manuales (III + IV).

c) Trabajadores manuales $(\mathrm{V}+\mathrm{VI}+\mathrm{VII})$.

Otro indicador complementario de clase social utilizado ha sido el nivel formativo de los padres, clasificado del siguiente modo: estudios universitarios, estudios postobligatorios y hasta estudios obligatorios.

Las variables que definen las características de los centros son dos. La primera es el grado de comprensividad, entendida como agrupación heterogénea u homogénea de alumnos en función de su nivel de rendimiento. Hay dos aproximaciones para medir el grado de comprensividad. La primera es a partir de la información subjetiva recogida de los equipos directivos sobre la organi-

6. En los casos donde solo disponemos de la puntuación de una prueba, se ha asignado dicho valor como indicador de rendimiento.

7. Según el criterio de dominancia: el nivel más alto de los dos progenitores.

8. International Standard Classification of Ocupations. 
Tabla 3. Clasificación de centros según la proporción de inmigración y de familias con ocupaciones manuales (alumnos de $4 .{ }^{\circ}$ )

\begin{tabular}{|c|c|c|c|c|c|}
\hline & \multicolumn{5}{|c|}{ Proporción de trabajadores manuales } \\
\hline & & $>29 \%$ & $11-29 \%$ & $0-10 \%$ & Tota \\
\hline \multirow{4}{*}{$\begin{array}{l}\text { Proporción } \\
\text { inmigrantes }\end{array}$} & $>39 \%$ & 5 & 2 & & 7 \\
\hline & $11-39 \%$ & & 4 & 2 & 6 \\
\hline & $0-10 \%$ & 1 & 1 & 12 & 14 \\
\hline & Total & 6 & 7 & 14 & 27 \\
\hline
\end{tabular}

Fuente: elaboración propia a partir de datos del ISCY Project.

zación del currículo. El problema de esta aproximación es que existe mucha diversidad de matices y algunas incoherencias en las declaraciones de los equipos directivos, por lo que no es fácil construir una tipología dicotómica entre centros comprensivos y centros no comprensivos (Merino et al, 2017). Esta dificultad en la definición misma de comprensividad tiene consecuencias para la política educativa, como se verá en las conclusiones.

La segunda aproximación es objetiva y se ha construido a partir de las diferencias entre las medias de la prueba de competencias básicas (PCB de aquí en adelante) entre los distintos grupos de $4 .^{\circ}$ de ESO en un mismo centro escolar. Aquí la suposición es que, si un centro tiene grupos heterogéneos, las diferencias de resultados en las PCB de los distintos grupos deberían ser mínimas o tendentes a cero ${ }^{9}$. En cambio, si los distintos grupos de $4 .^{\circ}$ de ESO están configurados en función del nivel de rendimiento de los alumnos, es de suponer que las diferencias de resultados en las PCB serán mayores, es decir, las distancias entre los grupos serán más grandes ${ }^{10}$.

La segunda característica de los centros es la composición social del alumnado. Como ya se ha mencionado anteriormente, la muestra del estudio se realizó teniendo en cuenta un criterio llamado de complejidad, establecido en función del porcentaje de alumnado con necesidades educativas especiales y en función del nivel de renta del entorno del centro escolar. Pero el hecho de que variables de entorno socioeconómico tuvieran un peso específico en la definición de la complejidad introduce un sesgo provocado por la segregación urbana, y por este motivo se ha construido un indicador de complejidad a partir de los datos obtenidos en la encuesta a los estudiantes ${ }^{11}$. Este indicador ha sido creado a partir del porcentaje de alumnos de familias con ocupaciones manuales y de alumnos de origen inmigrante, como se puede apreciar en la tabla 3.

9. Los tres centros con un solo grupo han sido clasificados como comprensivos.

10. Utilizamos un criterio cualitativo para determinar si las diferencias eran importantes, considerando además elementos que identificamos durante el trabajo de campo.

11. Esta opción también presenta un sesgo, ya que no todos los alumnos de los centros tuvieron el permiso paterno para contestar la encuesta, y se centra en los alumnos de $4 .^{\circ}$ de ESO, no de todos los alumnos del centro. El primer sesgo es difícilmente controlable, y el segundo, al tratarse de un estudio centrado en alumnos de $4 .^{\circ}$, parece razonable que sea asumible. 
El establecimiento de los límites de los intervalos de la tabla 3, tanto para la proporción de alumnos de origen inmigrante como de clase social trabajadora, se ha realizado teniendo en cuenta que los distintos grupos tengan un peso cuantitativo equilibrado. Así, la tipología que hemos construido es la de centros de alta complejidad ( $>10 \%$ de alumnos con padres trabajadores manuales y $>39 \%$ de alumnos de origen inmigrante; 7 centros destacados con sombreado oscuro); complejidad media ( $<30 \%$ de padres trabajadores manuales, entre un 11 y un $39 \%$ de inmigrantes, $>29 \%$ de padres trabajadores manuales y $<11 \%$ de inmigrantes; 7 centros destacados con sombreado claro), y baja complejidad ( $<30 \%$ de padres trabajadores y $<11 \%$ de inmigrantes; 13 centros sin sombrear).

Respecto a las técnicas de análisis, se han utilizado tablas de contingencia y comparación de medias para el análisis bivariado. Para el análisis multivariante, hemos utilizado el análisis lineal jerárquico, recogiendo variables de nivel individual y de centro como efectos fijos. La aproximación jerárquica o multinivel nos permite analizar la relación entre el individuo y el contexto en el que actúa, además, permite evaluar la importancia de las características del centro en relación con los factores individuales (Andreu, 2011). Hay que advertir que, al utilizar variables categóricas (tipología de centros, origen social y sexo) como predictoras de una variable continua (PCB), el ajuste y los coeficientes siempre tenderán a ser débiles.

En la tabla del anexo 1 se recogen algunos de los modelos realizados, así como algunos indicadores de capacidad explicativa y de ajuste. A partir del coeficiente de correlación intraclase (CCI) identificamos el porcentaje de varianza del rendimiento que corresponde al nivel de los centros y observamos su modificación al introducir los distintos factores en el modelo (Pardo et al., 2007). La comparación entre categorías se realiza mediante la corrección de Bonferroni (más estricta).

Antes de pasar a los resultados, hay que comentar otras limitaciones del modelo de análisis. La reducción de la diversidad social y de los centros de enseñanza secundaria a tipologías dicotómicas o de tres categorías es probablemente un ejercicio necesario para hacer el análisis inteligible, pero a la vez constituye un ejercicio de simplificación de esa complejidad social, casi una reducción a tipos ideales weberianos que son una abstracción de la realidad. En segundo lugar, el análisis basado en correlaciones y/o en varianzas muestra relaciones entre variables, relaciones que hay que interpretar en base a los mecanismos causales definidos en el marco teórico o, en su defecto, en base a explicaciones ad hoc.

En tercer lugar, nos hubiera gustado tener una variable diferente de rendimiento para medir la aptitud, e incluso disponer de datos acerca de los alumnos antes de acceder a la secundaria para establecer una relación más nítida entre las competencias que muestran los adolescentes antes y después de una etapa de escolarización tan importante como la secundaria obligatoria.

La primera y la segunda limitaciones quedarán reducidas en un futuro por la aportación de técnicas cualitativas en la investigación en curso, que comple- 
tará la comprensión de la relación entre clase, centro y adolescente. La tercera limitación tiene difícil solución, ya que no puede proyectarse el pasado para estimar las competencias del alumno antes de la educación secundaria, y por el hecho que el diseño de la investigación (que recordemos, está consensuado a nivel internacional) puso el inicio en el momento en que los individuos de la muestra tenían 15 años y estaban en el $100^{\circ}$ grado de sus estudios. Aún así, nos parece que el modelo aporta algunas novedades interesantes al debate sobre las desigualdades de resultados educativos.

\section{Análisis de resultados}

En la tabla 4 se ilustran algunos estadísticos descriptivos y un análisis bivariado para explorar el comportamiento de las variables introducidas respecto al rendimiento. Destaca la inexistencia de asociación del sexo con el rendimiento, debido a que el indicador utilizado recoge una media entre lengua y matemáticas, por lo que las diferencias entre chicos y chicas se compensan ${ }^{12}$.

Tabla 4. Estadísticos descriptivos de las variables utilizadas

\begin{tabular}{lrcc}
\hline Variables de estudiante & \multicolumn{1}{c}{$\%(\mathrm{~N})$} & Media PCB & d. t. \\
\hline Sexo & $100(1.761)$ & & \\
Chicos & $51,7(911)$ & 75,4 & 15,1 \\
Chicas & $48,3(850)$ & 74,4 & 14,7 \\
\hline Categoría profesional familiar & $100(1.705)$ & & \\
Empresarios y profesionales & $56,4(962)$ & $79,3^{\star}$ & 12,1 \\
Trabajadores no manuales & $30,1(512)$ & $71,7^{\star}$ & 15,2 \\
Trabajadores manuales & $13,5(231)$ & $65,1^{*}$ & 16,9 \\
\hline Nivel formativo familiar & $100(1.739)$ & & \\
Estudios universitarios & $50,5(878)$ & $79,2^{\star}$ & 12,4 \\
Estudios postobligatorios & $33,6(584)$ & $73,5^{\star}$ & 14,3 \\
Hasta estudios obligatorios & $15,9(277)$ & $64,6^{*}$ & 17,6 \\
\hline Variables de centro & $100(1.782)$ & & \\
Modelo de agregación & $46,1(821)$ & $78,5^{\star}$ & 11,4 \\
Comprensivo (13 centros) & $53,9(961)$ & $71,7^{\star}$ & 16,8 \\
Segregado (14 centros) & $100(1.782)$ & & \\
\hline Composición social & $20,4(363)$ & $61,9^{\star}$ & 18,5 \\
Alta complejidad (7 centros) & $25,7(458)$ & $74,7^{*}$ & 12,8 \\
Complejidad media (7 centros) & $53,9(961)$ & $79,6^{\star}$ & 11,2 \\
Baja complejidad (13 centros) & 1.782 & 74,9 & 14,9 \\
\hline Total &
\end{tabular}

* Diferencias significativas a $p$-value $<0,05$ (ANOVA).

Fuente: elaboración propia a partir de datos del ISCY Project.

12. No es objeto de este artículo analizar las desigualdades de rendimiento entre chicos y chicas, sino si existe diferencia en el efecto composición en ellos y ellas, por eso se ha utilizado la media de las pruebas de matemáticas y de lengua. 
La diferencia en el rendimiento existente entre los institutos de la muestra recoge, a partir de un modelo sin tener en cuenta las características individuales ni las características de centro (tabla anexo 1, modelo 2), cerca del 30\% de la variabilidad de las notas de PCB de los alumnos de 4. ${ }^{\circ}$ de ESO. Apunta así a la importancia de los efectos del centro más allá de los elementos individuales en el rendimiento. Las diferencias de rendimiento de los jóvenes según el centro escolar se hacen patentes con nuestros datos.

La cuestión clave a dilucidar es si estas diferencias se deben a un efecto composición o a un efecto centro. El primero respondería a la distinta proporción de estudiantes de extracción social diferente, con lo cual la comparación entre los centros no se produciría en igualdad de condiciones. El segundo efecto sería el resultado del modelo organizativo y/o pedagógico de los centros, que favorecería o desincentivaría el rendimiento del alumnado. Por último, tal como apunta algún autor (Carabaña, 2016), también se puede distinguir el efecto pares del efecto composición, según el cual la composición social del centro ejerce un efecto positivo o negativo entre los estudiantes más allá de las características individuales, sobre todo en los que están en una posición minoritaria debido a las dinámicas y a las relaciones que se producen en la institución docente. En los siguientes apartados se analizará cada uno de estos efectos.

\section{a) Clase social, sexo y rendimiento}

El primer elemento que contrastamos es el de las características individuales de los alumnos, concretamente el origen social y el sexo. Del 30\% de la varianza producida entre los distintos centros, al añadir estas variables, el porcentaje se reduce hasta aproximadamente el 21\% (tabla anexo 1, modelo 3). Esto significa que, aún controlando estas características individuales, las diferencias entre centros explican una quinta parte de la variabilidad de las PCB. Y lo que es más relevante aún: los centros recogen una mayor variabilidad de rendimiento, incluso controlada por el origen social y el sexo de cada alumno, que estos dos últimos factores individuales. Sin duda intervienen otras características personales, como pueden ser las prácticas familiares o el idioma materno, aunque aquí nos centramos en estos dos ${ }^{13}$. Ello nos remite a la importancia del debate sobre el papel de los centros escolares en el rendimiento que se aborda en dichos análisis.

Observamos además que el origen social influye en el rendimiento especialmente para el caso de los chicos, ya que las chicas de clase baja presentan un rendimiento significativamente mayor que los chicos de su mismo estatus social (gráfico 1). Así pues, las diferencias de clase para las chicas se reducen notablemente e incluso desaparecen estadísticamente. Las explicaciones a este fenómeno pueden buscarse en una mayor predisposición al estudio y/o en una mejor adaptación al entorno académico por parte de las chicas (o su rever-

13. Si el lector está interesado en el tema, se está preparando otra publicación en la que se analizan otros factores individuales con los mismos datos. 
Gráfico 1. Estimaciones de la media de PCB según la relación con el sexo y el origen social en el rendimiento educativo

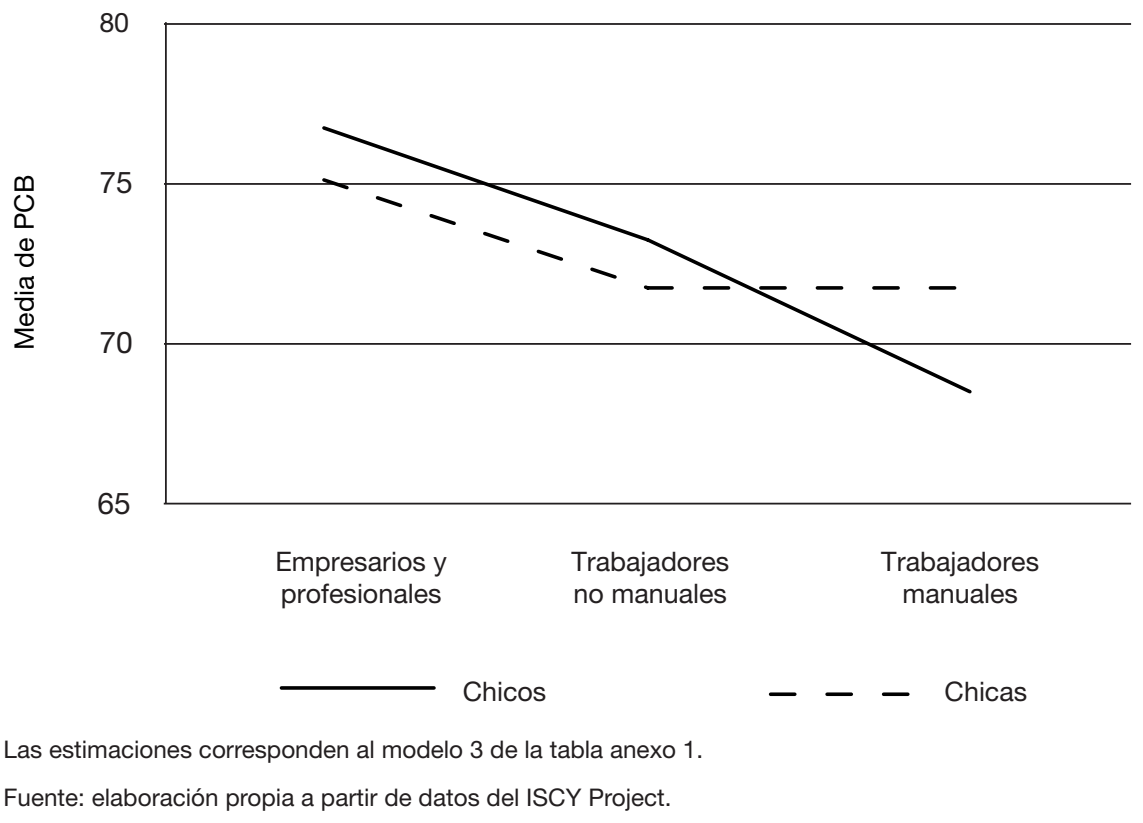

so: una mayor predisposición a la disrupción escolar por parte de los chicos de clase baja, debido a la socialización diferencial) o bien a un mecanismo de compensación ante les peores perspectivas futuras de inserción socioprofesional y/o de mercado matrimonial (Merle, 2009; Gambetta, 1987).

En conjunto, el efecto del origen social y del sexo explican solamente alrededor del $10 \%$ de la varianza intracentros. Es un porcentaje parecido a los que se han encontrado en otros trabajos (Carabaña, 2012).

Partiendo de este $21 \%$ de variabilidad intercentros una vez controladas algunas características individuales, nuestro objetivo es identificar hasta qué punto intervienen dos características del centro que, como hemos visto, están muy presentes en el debate educativo: la práctica o no de un modelo comprensivo y el efecto pares derivado de la composición del alumnado de cada institución docente.

\section{b) El modelo de agregación del alumnado: la comprensividad}

Una vez introducimos el tipo de agregación curricular entre alumnos en el modelo, observamos que del $21 \%$ de la variabilidad correspondiente a las diferencias entre centros, 3,3 puntos porcentuales corresponden a la utilización o no de un modelo comprensivo (que representa el 16\%, con lo que queda, pues, el $84 \%$ de la diferencia de centros restante aún sin explicar) (tabla anexo 
Tabla 5. Resultados del modelo lineal jerárquico de los efectos de la comprensividad en el rendimiento

\begin{tabular}{lcc}
\hline & Coeficiente & Error típico \\
\hline Constante & $79,88^{\star}$ & 1,88 \\
Categoría profesional de los padres: trabajadores manuales & $-8,19^{\star}$ & 1,48 \\
Categoría profesional de los padres: trabajadores no manuales & $-3,47^{\star}$ & 1,05 \\
Chicas hijas de trabajadores manuales & $5,01^{*}$ & 2,09 \\
Centros que segregan & $-6,02^{*}$ & 2,53 \\
\hline$N$ & \multicolumn{2}{c}{1.610} \\
-2LL & \multicolumn{2}{c}{$12.747,67$} \\
\hline
\end{tabular}

El resto de interacciones han sido descartadas, al no aportar una reducción de varianza significativa al modelo.

* Solo se muestran los coeficientes del modelo significativos al $<0,05$.

Fuente: elaboración propia a partir de datos del ISCY Project.

1, modelo 4). Es un porcentaje significativo, pero relativamente bajo, lo que apunta a la poca influencia de este elemento organizativo en el rendimiento de los alumnos, tal y como Coleman et al. (1966) ya advirtieron en su trabajo.

El alumnado con padres trabajadores manuales obtiene de media 8 puntos menos que el de padres empresarios o profesionales. Los resultados de la tabla 5 muestran, además, que son los centros más comprensivos los que obtienen en general mayores puntuaciones en comparación con los centros que segregan a sus alumnos por capacidad, con hasta 6 puntos de media de diferencia. Así mismo, no identificamos una interacción significativa de ello con el origen social. Así pues, el rendimiento en los centros comprensivos aumenta en general y para todas las clases sociales. Asistir a una escuela comprensiva sería una ventaja en términos de rendimiento para todas las posiciones sociales, teniendo en cuenta, además, que el colectivo de chicas de origen social bajo se comporta de forma diferente a lo esperable, puesto que ellas obtienen un rendimiento mayor (hasta 5 puntos de media) independientemente de la comprensividad del centro.

Aunque no es descartable el efecto de la comprensividad, hay que remarcar que no lo podemos identificar como un factor con mucho peso en comparación con los otros factores analizados. Por último, hay que decir que encontramos unos resultados parecidos si consideramos el nivel formativo familiar como variable de origen social ${ }^{14}$. Estos resultados contrastan con los obtenidos por Glaeser y Cooper (2012), ya que en nuestro caso es en los centros no selectivos donde se produce una mejora del rendimiento para todos los orígenes sociales.

\section{c) La composición social del alumnado: la complejidad}

Mucho más determinante es la composición del alumnado en la institución docente. De los 21 puntos porcentuales de la varianza intercentros que obser-

14. No se muestran por redundantes. 
Gráfico 2. Estimaciones de la media de PCB según la relación entre la composición social del centro y el origen social del alumnado

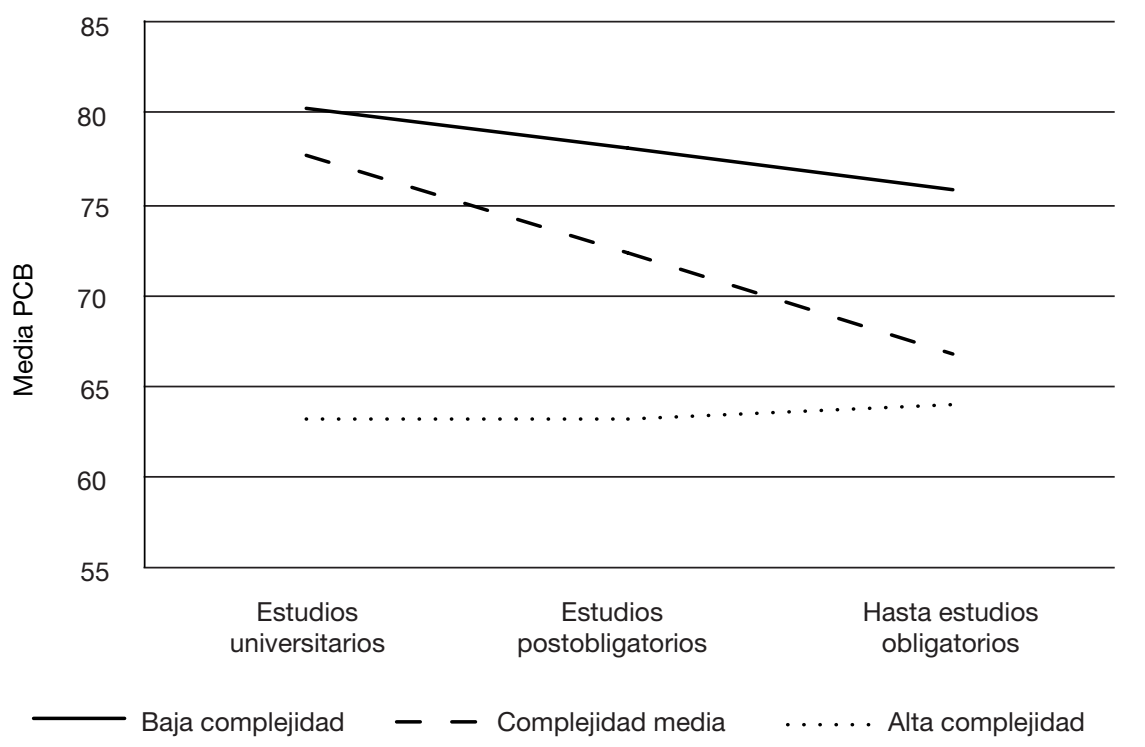

Fuente: elaboración propia a partir de datos del ISCY Project.

vamos, 13,5 estarían vinculados a la composición social (es decir, el 64\% de las diferencias intercentros). Sucede incluso que la composición de los centros da cuenta de una mayor variabilidad del rendimiento que los factores intracentros introducidos en el modelo (tabla anexo 1, modelo 5), lo que deja entrever un efecto pares. Las diferencias entre centros de baja y alta complejidad llega hasta los 14 puntos de media en las PCB, incluso controlando el origen social y el sexo de los alumnos (tabla 6).

Los resultados muestran que es en los centros de complejidad media donde se producen diferencias en el rendimiento según el origen social del alumno, puesto que el que obtienen los estudiantes de origen social bajo es más bajo. En este sentido, los estudiantes de centros con baja complejidad obtienen mejores resultados que el resto, sean del origen social que sean. Y lo mismo sucede pero en el sentido contrario con los alumnos de centros con alta complejidad (gráfico 2).

La interpretación de todo ello es que en centros en los que los alumnos de familias trabajadoras manuales son minoritarios, estos obtienen unos resultados parecidos al resto de sus compañeros cuyas familias se encuentran en una posición social más alta. El efecto pares ejercería una influencia positiva en ellos debido a la interacción dominante del grupo mayoritario por encima del grupo minoritario, o bien las familias de clase trabajadora podrían estar matriculando a sus hijos de mayor aptitud a estos centros en una suerte de proceso autose- 
Tabla 6. Resultados del modelo lineal jerárquico de los efectos de la complejidad en el rendimiento

\begin{tabular}{lcc}
\hline & Coeficiente & Error típico \\
\hline Constante & $81,04^{*}$ & 1,34 \\
$\begin{array}{l}\text { Chicas hijas de trabajadores manuales en centros de alta } \\
\quad \text { complejidad }\end{array}$ & $8,73^{*}$ & 2,59 \\
Alumnado con padres trabajadores manuales en centros & $-9,08^{*}$ & \multirow{2}{*}{4,19} \\
$\quad$ de complejidad media & $-13,71^{*}$ & 3,39 \\
Centro de alta complejidad & \multicolumn{2}{c}{1.610} \\
$N$ & \multicolumn{2}{c}{$12.645,49$} \\
\hline-2 LL & & \\
El resto de interacciones se han descartado, al no aportar una reducción de varianza significativa al \\
modelo. \\
* Solo se muestran los coeficientes del modelo significativos al <0,05. \\
Fuente: elaboración propia a partir de datos del ISCY Project.
\end{tabular}

lectivo, hipótesis que apuntan autores como Subirats (2008) o Bonal (2012a), entre otros, y que nuestros datos refuerzan. En cambio, en centros en los que los estudiantes de familias de profesionales son minoritarios, los resultados tienden a ser más bajos por las mismas razones.

Es en los centros más interclasistas donde se observan las diferencias esperadas entre alumnos según la clase social a la que pertenecen: hasta 9 puntos de media de diferencia entre hijos de profesionales e hijos de trabajadores manuales (tabla 6). Aún así, los hijos de trabajadores manuales obtienen mejor resultado en estos centros interclasistas que en los centros donde son mayoría. Como se retomará en el apartado de conclusiones, esta cuestión es fundamental para la gestión de la diversidad y las políticas contra la segregación escolar.

En la tabla 6 observamos de nuevo la interacción entre origen social y sexo. Como ya se ha señalado, las chicas de clase baja obtienen mejores resultados que los chicos de su mismo nivel social. Si se incorpora también la complejidad del centro se observa una diferencia favorable a las chicas aún mayor (casi 9 puntos más de media). Así pues, el mayor rendimiento académico de las hijas de trabajadores manuales que observábamos ya en el grafico 1 solamente se produce en centros de alta complejidad, es decir donde son mayoría. En otros centros donde no son mayoría, los resultados de esta clase social no varían por sexo.

Las chicas de clase baja que estudian en entornos escolares complejos muestran un rendimiento parecido al que obtendrían en otros contextos más favorables (hasta 9 puntos más de media), a diferencia de los chicos, que se ven más perjudicados. Dicho de otra forma, la complejidad del centro tiene mayor incidencia en ellos, ya que estarían más influenciados por el efecto pares (las dinámicas y las interacciones entre compañeros, el contagio de expectativas, etc.).

Por último, hay que remarcar que utilizando el nivel formativo familiar como indicador de origen social, los resultados varían respecto a lo comentado (tabla anexo 2): no se encuentran patrones significativamente distintos en las diferen- 
Gráfico 3. Estimaciones de la media de PCB según la relación entre la composición social del centro y el nivel formativo familiar del alumno

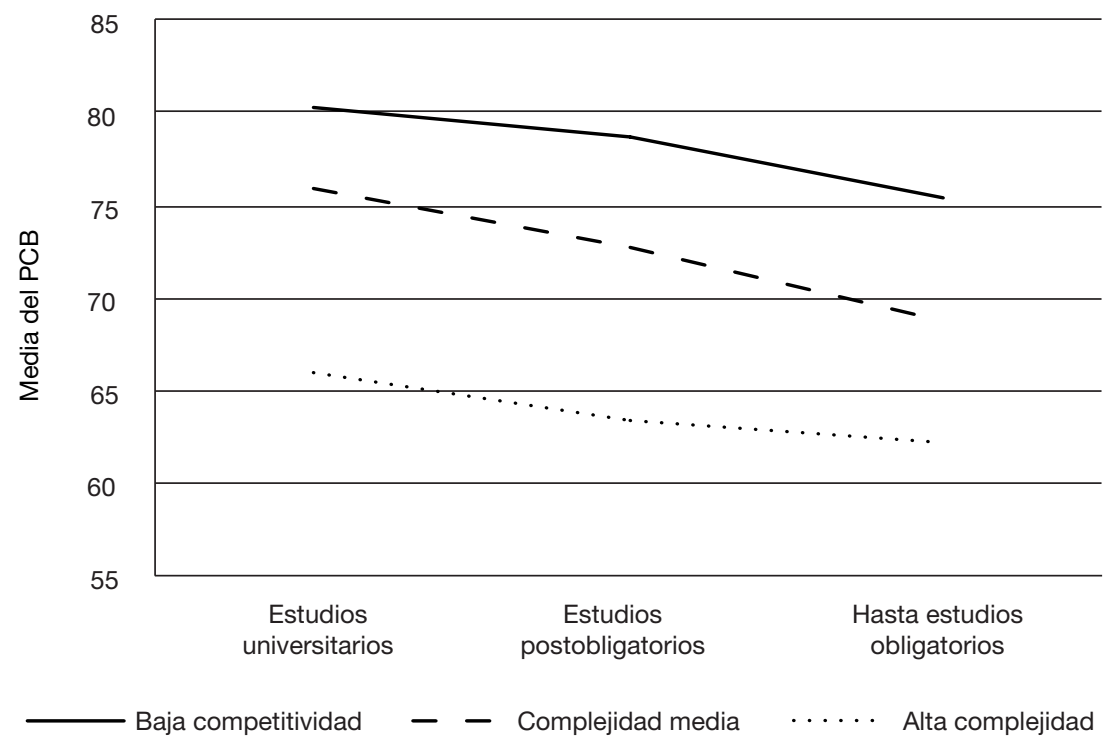

Fuente: elaboración propia a partir de datos del ISCY Project.

cias entre niveles formativos de los padres según el contexto escolar del alumno (gráfico 3). A diferencia de lo observado en el gráfico 2, los estudiantes con padres universitarios obtendrían mejor rendimiento aún en contextos de alta complejidad.

Esta diferencia de resultados basándose en la ocupación de los padres podría deberse al hecho de que el efecto del nivel formativo familiar, mucho más asociado a aspectos cognitivos y culturales, sea menos maleable por el efecto pares. Aunque no inmune, dado que la distancia con los hijos de padres universitarios en centros de baja complejidad es considerable. Por lo tanto, el efecto de la categoría profesional de los padres dependería más del contexto que el efecto del nivel formativo de los padres, que es más estable.

\section{d) La relación entre comprensividad y la complejidad de los centros}

Por último, ponemos en relación tanto el modelo de agregación del alumnado como la composición social del centro. Combinar las dos dimensiones no aporta al modelo una capacidad explicativa adicional, incluso la reduce, y tampoco reduce el CCI (tabla anexo 1, modelo 6). Por lo tanto, descartamos el modelo. De hecho, los efectos de la comprensividad desaparecen por completo del modelo al añadir la composición social del centro (tabla 7), tal y como Benito y González (2013) señalan también en su estudio y que matizan el de Glaeser y Cooper (2012).

Esto significa, por un lado, una elevada correlación entre ambas dimensiones: a menor complejidad del centro, mayor comprensividad ( $V$ de Cramer de 0,7). 
Tabla 7. Resultados del modelo lineal jerárquico de los efectos de la complejidad y de la comprensividad en el rendimiento

\begin{tabular}{|c|c|c|}
\hline Factores & $\mathrm{F}$ & Sig. \\
\hline Modelo corregido & 5,68 & * \\
\hline Categoría profesional familiar & 10,11 & * \\
\hline Sexo & 1,57 & ns \\
\hline Categoría profesional*sexo & 1,90 & ns \\
\hline Complejidad del centro & 9,81 & * \\
\hline Categoría profesional ${ }^{*}$ complejidad & 4,59 & * \\
\hline Comprensividad & 0,07 & ns \\
\hline Categoría profesional ${ }^{*}$ comprensividad & 0,01 & ns \\
\hline Categoría profesional*complejidad ${ }^{*}$ sexo & 2,11 & * \\
\hline Categoría profesional ${ }^{*}$ comprensividad ${ }^{\star}{ }^{\circ}$ complejidad & 0,88 & ns \\
\hline $\bar{N}$ & \multicolumn{2}{|c|}{1.610} \\
\hline$-2 \mathrm{LL}$ & \multicolumn{2}{|c|}{$12.623,72$} \\
\hline
\end{tabular}

* significativo a $p$-value $<0,05$. Ns: no significativo.

Fuente: elaboración propia a partir de datos del ISCY Project.

Dicho de otro modo, los centros de menor complejidad tienen más oportunidades para no utilizar grupos de nivel homogéneo, aunque algunos los utilicen. En cambio, los centros de alta complejidad lo tienen mucho más difícil, porque, aunque quisieran hacer grupos heterogéneos, la diversidad de rendimiento asociada a la diversidad social lo hace más complicado (Merino et al, 2017).

Por otro lado, significa una mayor importancia de la composición social en comparación con los elementos organizativos del centro, al menos evaluados en términos de comprensividad. Las relaciones constatadas en el punto anterior no varían, pues, en función del modelo de agregación del alumnado del centro.

\section{Conclusiones}

La principal conclusión de nuestro estudio es que, entre los factores que inciden en la desigualdad de resultados educativos, la segregación escolar (por el efecto composición y el efecto pares) tiene mayor capacidad explicativa de la desigualdad que el modelo organizativo de los centros. El alumnado que procede de familias trabajadoras se ve más desfavorecido si se escolariza en centros donde la clase trabajadora es mayoritaria, mientras que la organización curricular, tal y como la hemos definido en nuestra investigación, tiene un impacto muy pequeño en la mejora de resultados. Es decir, el efecto de la segregación escolar sobre la desigualdad de resultados es mayor que el hecho de estar en escuelas más selectivas o más comprensivas a nivel de prácticas pedagógicas.

Las implicaciones políticas de estos resultados parecen claras. Será más efectivo luchar contra la segregación académica que proponer reformas curriculares u organizativas, por lo menos en la última etapa de la ESO, pero es mucho más difícil, porque la segregación académica se sostiene en dos pilares estructurales. El primero 
es la segregación urbana, producto de políticas urbanísticas y de dinámicas sociales que fijan las desigualdades sociales en el territorio. Aunque existen experiencias exitosas que intentan limitar los efectos de la segregación urbana en la segregación escolar (Bonal, 2012b), el impacto no puede ser muy elevado e incluso a veces pueden provocar efectos no deseados. Un ejemplo es el busing, que pretendía disminuir la segregación racial después de las leyes de derechos civiles en Estados Unidos repartiendo a chicos afroamericanos por escuelas de clase media blanca, lo que provocó la White Flight (Gamoran y Long, 2006). El segundo pilar es precisamente lo que permitió esta huida, la elección de centro por parte de las familias, muy enraizada en sistemas liberales como en los Estados Unidos, pero también en parte consagrada por nuestro sistema normativo y en buena parte incorporada a las mentalidades y a las estrategias de movilidad social de los individuos (Erikson, 1996). El análisis de la elección de centro por parte de las familias queda fuera de nuestro propósito, pero apunta que existe una relación entre una mayor posibilidad de escoger y una menor equidad en el acceso y en los resultados educativos (OECD, 2011). Muchas familias están más preocupadas acerca de quiénes serán los compañeros de clase de sus hijos que del proyecto pedagógico del centro.

Las reformas comprensivas que se reduzcan a la gestión de las aulas, por muy bien intencionadas que estén, son de alcance limitado en los contextos de alta complejidad. Aún así, nuestros resultados fortalecen la idea de que una escuela comprensiva es mejor que una selectiva en términos de resultados (aunque poco) y para todas las clases sociales. También se pueden buscar otros argumentos a favor de la comprensividad que no se basen en la mejora de resultados, sino en la mejora de la gestión de la diversidad o del clima del aula y del centro (FerrerEsteban, 2015), para evitar la etnificación de los grupos de menor rendimiento (Pàmies y Castejón, 2015), o simplemente porque es un proyecto de interés para el profesorado. No podemos olvidar que nuestra operacionalización de la comprensividad parte del supuesto de que una agrupación por niveles produce mayor variación en el rendimiento medido por las pruebas externas de competencias básicas, y una agrupación heterogénea arrojaría resultados menos dispares entre los distintos grupos. Pero estos resultados pueden ser debidos a más de un modelo de comprensividad, con agrupaciones flexibles o desdoblamientos en algunas asignaturas u otras combinaciones posibles (Ferrer-Esteban, 2015).

Por último, hay un elemento relevante a destacar: las chicas de clase baja, las cuales, aún en centros de alta complejidad, obtienen resultados parecidos a los que alcanzan en centros de menor complejidad. Es posible, y lo apuntamos como hipótesis para futuras investigaciones, que el efecto de pares pueda compensar en parte el efecto de composición del centro, que las relaciones y las interacciones entre chicas refuerce su adaptación o su estrategia de éxito académico.

\section{Referencias bibliográficas}

ANDREU, Jaime (2011). «El análisis multinivel: Una revisión actualizada en el ámbito sociológico». Metodología de Encuestas, 13, 161-176. <http://casus.usal.es/pkp/index.php/MdE/article/view/1017>. 
BENITO, Ricardo y GONZÁleZ, Isaac (2013). «¡Puede el efecto composición reducir las desigualdades educativas?: Una mirada sobre los efectos de la segregación escolar en España y los países del modelo de integración uniforme». Revista de la Asociación de Sociología de la Educación, 6 (1), 49-71.

BONAL, Xavier (2012a). «Education policy and school segregation of migrant students in Catalonia: The politics of non-decision-making». Journal of Education Policy, 27 (3), 401-421. <http://dx.doi.org/10.1080/02680939.2011.645168>

BOnAL, Xavier (dir.) (2012b). Municipis contra la segregació escolar: Sis experiències de política educativa local. Barcelona: Fundació Bofill.

CABrera, Leopoldo y CABrera, Blas (2008). «Heterogeneidad de centros y desigualdad de rendimientos educativos». Témpora, 11, 61-99. $<$ http://dialnet.unirioja.es/servlet/oaiart?codigo $=3098540>$.

Calero, Jorge (2007). Desigualdades socioeconómicas en el sistema educativo español. Madrid: Ministerio de Educación y Ciencia / CIDE.

CARABAÑa, Julio (1993). «Sistema de enseñanza y clases sociales». En: García DE LeOn, M. a Antonia; Fuente, Gloria de la y OrtegA, Félix (eds.). Sociología de la Educación. Barcelona: Barcanova.

- (2012). «Debilidades de PISA y errores en la atribución del fracaso escolar académico». En: Puelles, Manuel (ed.). El fracaso escolar en el estado de las autonomías. Madrid: Wolters Kluwer.

- (2016). «El informe Coleman, 50 años después». Revista de la Asociación de Sociología de la Educación, 9 (1), 9-21.

Coleman, J. et al. (1966). Equality of educational opportunity. Washington: U. S. Government Printing Office.

Dupriez, Vincent; DumaY, Xavier y Vause, Anne (2008). «How do School Systems Manage Pupils' Heterogeneity?: A Reanalysis of PISA 2003». Comparative Education Review, 52 (2), 245-273. <http://dx.doi.org/10.1086/528764>

Duru-Bellat, Marie y Mingat, Alain (1997). «La constitution de classes de niveau dans les collèges: Les effets pervers d'une pratique à visée égalisatrice». Revue Française de Sociologie, 38 (4), 759-789. <http://dx.doi.org/10.2307/3322627>

ERIKSON, Robert (1996). «Explaining Change in Educational Inequality-Economic Security and School Reforms». En: ERIKSON et al. (eds.). Can education be equalized?: The Swedish case in comparative perspective. Boulder, Colorado: Westview.

ERIKSON, Robert y GoldTHORPE, John (1992). The Constant Flux. Oxford: Clarendon Press.

FERNÁNDEZ EnGUITA, Mariano (2014). «De la información al conocimiento... pero en serio». Participación Educativa, 3 (5), 51-59. $<$ http://ntic.educacion.es/cee/revista/pdfs/n5art_mariano_fernandezenguita.pdf>.

- (2016). «El Informe Coleman: Una lección de sociología y de política». Revista de la Asociación de Sociología de la Educación, 9 (19), 37-45.

FERRER, Ferran (dir.) (2011). PISA 2009: Avaluació de desigualtats educatives a Catalunya. Barcelona: Fundació Bofill. <http://www.fbofill.cat/publicacions/pisa2009-avaluacio-de-les-desigualtats-educatives-catalunya>.

FERrer-Esteban, Gerard (2015). «QQué estrategias de agrupamiento responden a criterios de efectividad y equidad?». En: ¿Qué funciona en educación?: Evidencias para la mejora educativa. Barcelona: Fundació Bofill. <http://www.fbofill.cat/sites/ default/files/Que_funciona\%20en\%20educacion_02_010116.pdf>. 
FORQUiN, Jean Claude (1985). «El enfoque sociológico del éxito y el fracaso escolares: Desigualdades de éxito escolar y origen social» Educación y Sociedad, 3, 198-228.

GAmBETTA, Diego (1987). Were they pushed or did they jump? Individual decision mechanisms in education. Cambridge: University Press. <http://dx.doi.org/10.1017/ CBO9780511735868>

Gamoran, Adam y Long, Daniel (2006). Equality of Educational Opportunity: A 40-Year Retrospective. WECR Working Paper 2006-9. Madison: University of Wisconsin-Madison. <http://www.wcer.wisc.edu/publications/workingPapers/papers.php>.

GlaEser, Judith y COOPER, Barry (2012). «Educational achievement in selective and comprehensive local education authorities: A configurational analysis». British Journal of Sociology of Education, 33 (2), 223-244. <http://dx.doi.org/10.1080/01425692.2011.649833>

HanusheK, Eric y Woessmann, Ludger (2006). «Does Educational Tracking Affect Performance and Inequality? Differences-in-Differences Evidence Across Countries». The Economic Journal, 116, 63-75.

HARRIS, Judith-Rich (1999). El mito de la educación. Barcelona: Grijalbo.

HatTIE, John (2009). Visible Learning: A Synthesis of over 800 meta-analuyses Relating to Achievement. Londres: Routledge.

JACKSON, Michelle et al. (2007). «Primary and Secondary Effects on Class Differentials in Educational Attainment: The Transition to A level Courses in England and Wales». Acta Sociologica, 50, 211-229.

JaCKSOn, Michelle (ed.) (2013). Determined to suceed: Performance versus choice in Educational Attainment. California: Standford University Press. <http://dx.doi.org/10.11126/stanford/9780804783026.001.0001>

Martín Criado, Enrique; Gómez, Carmuca; Fernández-Palomares, Fernando y Rodrígez-Monge, Ángel (2000). Familias de clase obrera y escuela. Donostia: Iralka.

MERINO, Rafael (2012). «Desigualtats socials i desigualtats educatives: Escola, reproducció i mobilitat social». En: RotGER, Josep Maria (ed.). Sociologia de l'educació per a professorat de secundària. Barcelona: El Roure.

MERINO, Rafael et al. (2017). «Separación del alumnado por niveles en $4^{\circ}$ de ESO y complejidad social de los centros. Límites y posibilidades del currículum comprensivo". Témpora. Revista de sociología de la educación. 19, 181-198.

MERle, Pierre (2009). La démocratisation de l'enseignement. París: La Découverte.

OECD (2011). Equity and quality in Education: Suporting Disavantatged Students and Schools. París: OECD Publishing. <http://dx.doi.org/10.1787/9789264130852-en>

- (2013). PISA 2012 Results: What Makes Schools Successful? Resources, Policies and Practices, IV. París: OECD Publishing. <http://dx.doi.org/10.1787/9789264201156-en>

PÀMIES, Jordi y CASTEJÓN, Alba (2015). «Distribuyendo oportunidades: El impacto de los agrupamientos escolares en la experiencia de los estudiantes». Revista de la Asociación de Sociología de la Educación, 8 (3), 335-348.

PArDo, Antonio; Ruiz, Miguel Ángel y SAN MARTín, Rafael (2007). «Cómo ajustar e interpretar modelos multinivel con SPSS». Psicothema, 19 (2), 308-321.

SLAVIN, Robert (1990). "Achievement Effects of Ability Grouping in Secondary Schools: A Best-Evidence Synthesis». Review of Educational Research, 60 (3), 471-499. <http://dx.doi.org/10.3102/00346543060003471> 
SUBIRATS, Joan (2008). Efectes de la composició social dels centres d'ensenyament obligatori sobre el rendiment $i$ les expectatives de l'alumnat. Barcelona: IGOP.

TroiAnO, Helena y Elias, Marina (2014). «University access and after: Explaining the social composition of degree programmes and the contrasting expectations of students». Higher Education, 67 (5), 637-654.

<http://doi.org/10.1007/s10734-013-9670-4>.

\section{Anexo}

Tabla anexo 1. Resumen de la capacidad explicativa y ajustes globales de los modelos realizados

\begin{tabular}{|c|c|c|c|c|c|}
\hline & \multicolumn{5}{|c|}{ Varianza de PCB } \\
\hline & BIC & $\mathrm{CCl}$ & $\begin{array}{l}\text { Explicada } \\
\text { intracentros }\end{array}$ & $\begin{array}{l}\text { Explicada } \\
\text { intercentros }\end{array}$ & $\begin{array}{l}\text { No explicada } \\
\text { intercentros }\end{array}$ \\
\hline M1. Variables individuales & $12.997,15$ & - & $15,7 \%$ & - & - \\
\hline M2. Controlando por centro & $13.542,67$ & 0,28 & $0 \%$ & $0 \%$ & $28,8 \%$ \\
\hline $\begin{array}{l}\text { M3. Variables individuales } \\
\text { y controlando por centro }\end{array}$ & $12.771,44$ & 0,20 & $9,2 \%$ & $0 \%$ & $20,6 \%$ \\
\hline $\begin{array}{l}\text { M4. Variables individuales, } \\
\text { controlando por centro } \\
\text { y tipo de comprensividad }\end{array}$ & $12.740,06$ & 0,20 & $9,2 \%$ & $3,3 \%$ & $17,3 \%$ \\
\hline $\begin{array}{l}\text { M5. Variables individuales, } \\
\text { controlando por centro } \\
\text { y composición }\end{array}$ & $12.669,23$ & 0,20 & $9,9 \%$ & $13,2 \%$ & $7,4 \%$ \\
\hline $\begin{array}{l}\text { M6. Variables individuales, } \\
\text { controlando por centro, } \\
\text { comprensividad y composición }\end{array}$ & $12.638,91$ & 0,20 & $9,8 \%$ & $12.8 \%$ & $7,8 \%$ \\
\hline
\end{tabular}

$\mathrm{CCl}$ : coeficiente de correlación intraclase. Proporción de varianza intercentros respecto a la varianza intracentros.

BIC: criterio de información bayesiano.

Fuente: elaboración propia a partir de datos del ISCY Project.

Tabla anexo 2. Resultados del modelo lineal de los efectos de la complejidad en el rendimiento según el nivel formativo familiar

\begin{tabular}{lcc}
\hline & Coeficiente & Error típico \\
\hline Constante & $81,43^{*}$ & 1,34 \\
Padres con estudios obligatorios & $-7,71^{\star}$ & 2,92 \\
Centro de alta complejidad & $-13,43^{\star}$ & 3,46 \\
\hline$N$ & \multicolumn{2}{c}{1.639} \\
-2 LL & \multicolumn{2}{c}{$12.941,75$} \\
\hline
\end{tabular}

El resto de interacciones se han descartado, al no aportar una reducción de varianza significativa al modelo.

Solo se muestran los coeficientes del modelo significativos al <0,05.

Fuente: elaboración propia a partir de datos de ISCY Project. 